\title{
Effect of age on upper and lower eyelid saccades
}

\section{L.V.O. Leite ${ }^{1}$, A.A.V. Cruz ${ }^{1}$, A. Messias ${ }^{1}$ and J.M.C. Malbouisson ${ }^{2}$}

\author{
'Departamento de Oftalmologia, Otorrinolaringologia, \\ Cirurgia de Cabeça e Pescoço, Faculdade de Medicina de Ribeirão Preto, \\ Universidade de São Paulo, Ribeirão Preto, SP, Brasil \\ ${ }^{2}$ Departamento de Física Geral, Instituto de Física, \\ Universidade Federal da Bahia, Salvador, BA, Brasil
}

\section{Correspondence \\ A.A.V. Cruz \\ Departamento de Oftalmologia, \\ Otorrinolaringologia, Cirurgia \\ de Cabeça e Pescoço \\ HF, FMRP, USP \\ Av. Bandeirantes, 3900 \\ 14049-900 Ribeirão Preto, SP \\ Brasil \\ E-mail: aavecruz@fmrp.usp.br \\ Research supported by CNPq. \\ Publication supported by FAPESP.}

Received March 6, 2006

Accepted September 11, 2006

\begin{abstract}
To study the effect of age on the metrics of upper and lower eyelid saccades, eyelid movement of two groups of 30 subjects each were measured using computed image analysis. The patients were divided on the basis of age into a younger group (20-30 years) and an older group (60-91 years). Eyelid saccade functions were fitted by the damped harmonic oscillator model. Amplitude and peak velocity were used to compare the effect of age on the saccades of the upper and lower eyelid. There was no statistically significant difference in saccade amplitude between groups for the upper eyelid (mean \pm SEM; upward, young $=9.18 \pm 0.32 \mathrm{~mm}$, older $=8.93 \pm 0.31 \mathrm{~mm}, \mathrm{t}=0.56$, $\mathrm{P}=0.58$; downward, young $=9.11 \pm 0.27 \mathrm{~mm}$, older $=8.86 \pm 0.32$ $\mathrm{mm}, \mathrm{t}=0.58, \mathrm{P}=0.56$ ) However, there was a clear decline in the peak velocity of the upper eyelid saccades of older subjects (upward, young $=59.06 \pm 2.34 \mathrm{~mm} / \mathrm{s}$, older $=50.12 \pm 1.95 \mathrm{~mm} / \mathrm{s}, \mathrm{t}=2.93, \mathrm{P}=0.005$; downward, young $=71.78 \pm 1.78 \mathrm{~mm} / \mathrm{s}$, older $=60.29 \pm 2.62 \mathrm{~mm} / \mathrm{s}$, $\mathrm{t}=3.63, \mathrm{P}=0.0006$ ). In contrast, for the lower eyelid there was a clear increase of saccade amplitude in the elderly group (upward, young = $2.27 \pm 0.09 \mathrm{~mm}$, older $=2.98 \pm 0.15 \mathrm{~mm}, \mathrm{t}=4.33, \mathrm{P}<0.0001$; downward, young $=2.21 \pm 0.10 \mathrm{~mm}$, older $=2.96 \pm 0.17 \mathrm{~mm}, \mathrm{t}=3.85$, $\mathrm{P}<0.001)$. These data suggest that the aging process affects the metrics of the lid saccades in a different manner according to the eyelid. In the upper eyelid the lower tension exerted by a weak aponeurosis is reflected only on the peak velocity of the saccades. In the lower eyelid, age is accompanied by an increase in saccade amplitude which indicates that the force transmission to the lid is not affected in the elderly.
\end{abstract}

\section{Introduction}

When the gaze shifts from the primary position to up- or downgaze the eyelids also move accompanying the direction of the eye rotation. These eyelid movements are named eyelid saccades because their metrics closely
Key words

- Upper eyelid saccade

- Lower eyelid saccade

- Aging

- Digital image processing 
The eyelid mechanics are also affected by aging. In the upper eyelid, aponeurotic ptosis is a common finding (3) and in the lower eyelid horizontal laxity due to stretching of the canthal tendons can be demonstrated in the majority of elderly patients (4).

Very few studies have described the effect of aging on eyelid saccades. We were able to find only one article with data for upper eyelid saccades in young and older subjects (5). The effect of aging on the lower eyelid saccades has not been well documented. The few data on the amplitude of lower eyelid saccades have been measured with rulers (6), a procedure that does not allow a precise description of the effect of age on the metrics of these movements.

We have recently demonstrated that both upper and lower eyelid saccades can be accurately measured with a commercial video camera and mathematically analyzed with a model extensively used in engineering to describe elastic movements (damped harmonic oscillator) (7).

In the present study, we measured the upper and lower eyelid saccades of young and old normal subjects to determine the effect of aging.

\section{Subjects, Material and Methods}

\section{Subjects}

Sixty healthy subjects without history of eye or eyelid surgery were included in the study. They were divided into two groups of 30 each according to their age. Group A consisted of subjects (10 males and 20 females) aged 20 to 30 years (mean $\pm \mathrm{SD}=$ $26.33 \pm 3.11$ ) and group B consisted of older subjects (13 males and 17 females) aged 60 to 91 years (mean $\pm \mathrm{SD}=70.63 \pm 8.28$ )

None of the subjects showed any sign of ophthalmic or neurological abnormality except for a moderate degree of cataract in the older group.

Written informed consent was obtained from all subjects and the study was approved by the institutional Ethics Committee.

\section{Procedure}

First, the palpebral fissure of all subjects was photographed in the primary position of gaze with a digital camera. The images were then transferred to a microcomputer and the Image $\mathbf{J}$ software (available at http://rsb.info. nih.gov/ij/) was used to measure the midpupileyelid distance of the upper and lower eyelids.

The saccades of the upper and lower eyelids were then measured. Only one eye randomly chosen was used. The methods employed for this measurement have been described in detail elsewhere (7). Briefly, with their heads stabilized on a chin rest, the subjects were instructed to look down and up between two targets $20^{\circ}$ above and below a central light that was aligned with the subject's visual axis in the primary position of gaze ( $40^{\circ}$ of eye rotation across the upper and lower hemifields).

Eyelid motion during downward and upward eyelid saccades was recorded with a coupled-charged device (CCD) camera connected to a personal computer by a frame grabber. The temporal resolution of the camera was the standard NTSC $(30 \mathrm{~Hz}$ or 30 frames per second). Movement was analyzed with a software which calculated in real time the center of a blue spot in each frame. In order to provide the signal for the software a small piece of blue paper was attached to the eyelashes of the central portion of the upper eyelid. A similar spot was made on the skin of the lower eyelid margin with blue make-up.

\section{Results}

Figure 1 displays the mid-pupil eyelid distance distributions of the upper and lower eyelids. The mean mid-pupil eyelid distance of both eyelids was significantly smaller in the older group than in the young group (mean \pm 
SD; upper eyelid: young $=3.77 \pm 0.73$, older $=$ $2.97 \pm 0.95 \mathrm{~mm}, \mathrm{t}=3.68, \mathrm{P}=0.0005$; lower eyelid: young $=6.45 \pm 0.69$, older $=5.05 \pm$ $1.10 \mathrm{~mm}, \mathrm{P}<0.00001$ ) indicating a clear tendency for the eyelids to rest close to the pupil center in the elderly population.

The mean values of the saccade parameters for the downward and upward saccades of the two groups of subjects are shown in Table 1 (upper eyelid and lower eyelid). For the upper eyelid there was no difference in amplitude between the saccades of the older and young subjects (independent $t$-test, upward: $\mathrm{t}=0.56, \mathrm{P}=0.58$; downward: $\mathrm{t}=0.58$, $\mathrm{P}=0.56)$. However, the saccades of the older subjects were slower than the saccades of the young group (upward: $\mathrm{t}=2.93, \mathrm{P}=$ 0.005 ; downward: $\mathrm{t}=3.63, \mathrm{P}=0.0006$ ). The decrease in velocity demonstrated in the older subjects was not accompanied by any change in the functions relating amplitude and velocity to time (Figure 2). The linear relationship between amplitude and velocity shown in young subjects was also observed in older subjects (Figure 3).

Aging had a completely different effect on the dynamics of the lower eyelid. The saccade amplitude of the older group was significantly greater than the amplitude of the young (independent $t$-test, upward: $\mathrm{t}=$ 4.33, $\mathrm{P}<0.0001$; downward: $\mathrm{t}=3.85, \mathrm{P}<$ 0.001). Similarly to the upper eyelid, the relationships between amplitude and velocity and time were normal (Figure 4). A linear relationship was found between amplitude and velocity (Figure 5). When the velocity data were grouped (downward + upward, Figure 6) the mean velocity was also higher in the older group (young $=15.87$, old $=$ $17.85 \mathrm{~mm} / \mathrm{s}, \mathrm{t}=2.20, \mathrm{P}=0.03)$. When velocity was compared according to the direction of gaze (downward vs downward and upward $v s$ upward) the difference was not statistically significant.

\section{Discussion}

Aging induces a process of deterioration of collagen and other tissues resulting in the attenuation of the levator muscle aponeurosis in the upper eyelid (8). This process is the underlying cause of the aponeurotic ptosis. The decline in the mean mid-pupil eyelid distance verified here in the older group is consistent with this concept. From a mechanical perspective, weak aponeurosis

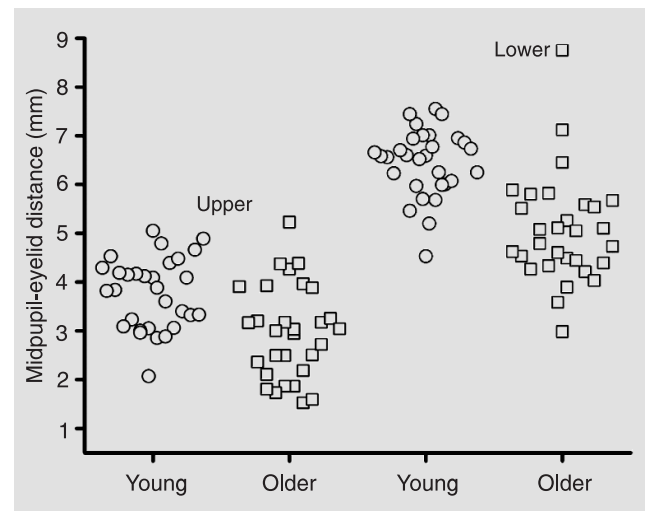

Figure 1. Mid-pupil eyelid distances for the upper and lower eyelid in young and older subjects.

Table 1. Parameters of upper and lower eyelid saccades

\begin{tabular}{lrrrrr}
\hline Parameter & \multicolumn{2}{c}{ Downward gaze } & & \multicolumn{2}{c}{ Upward gaze } \\
\cline { 2 - 3 } \cline { 5 - 6 } & \multicolumn{1}{c}{ Young } & Older & & Young & \multicolumn{1}{c}{ Older } \\
\hline Upper eyelid & & & & \\
$\quad$ Final amplitude $(\mathrm{mm})$ & $9.11 \pm 0.27$ & $8.86 \pm 0.32$ & & $9.18 \pm 0.32$ & $8.93 \pm 0.31$ \\
$\quad$ Peak velocity $(\mathrm{mm} / \mathrm{s})$ & $71.78 \pm 1.78$ & $60.29 \pm 2.62$ & & $59.06 \pm 2.34$ & $50.12 \pm 1.95$ \\
& & & & & \\
Lower eyelid & & & & & \\
$\quad$ Final amplitude $(\mathrm{mm})$ & $2.21 \pm 0.10$ & $2.96 \pm 0.17$ & & $2.27 \pm 0.09$ & $2.98 \pm 0.15$ \\
$\quad$ Peak velocity $(\mathrm{mm} / \mathrm{s})$ & $16.10 \pm 0.72$ & $18.34 \pm 1.15$ & & $15.72 \pm 0.76$ & $17.26 \pm 0.93$ \\
\hline
\end{tabular}

Data are reported as mean \pm SEM. 
Figure 2. Upper eyelid saccade amplitude: $A$, down; $C$, up. The evolution of velocity with time $(B$, down; $D$, up) is illustrated on the right. Note that, although the saccades of the older subjects are slower, they have essentially the same amplitude as the saccades of the young subjects.
Figure 3. Linear relationships between upper eyelid saccade amplitude and velocity for young (left, $r=0.71$ ) and older subjects (right, $r=0.75$ ). means less tension on the tarso-ligament system of the upper eyelid. It is interesting to note that the effect of this decrease in tension is reflected only in the velocity of the upper eyelid saccades. In elderly subjects, the functions relating upper eyelid position to time were shifted to the right but otherwise were essentially normal. The decline of the peak velocity of the upper eyelid saccades with age has been reported by Wouters et al. (5).

During vertical eye saccades, force transmission to the lower eyelid is believed to occur through the lower retractors (9). These are formed by two layers: the capsulopalpebral fascia and the inferior tarsal muscle. In the terminal portion of the retractors only the capsulopalpebral fascia is detected. This fascia is attached to the inferior tarsal border
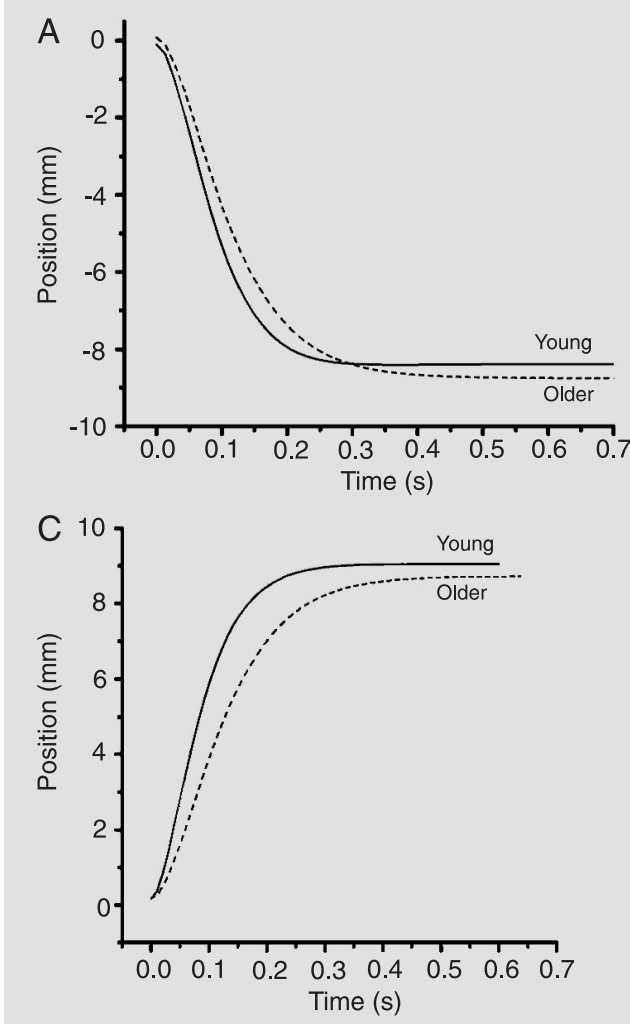
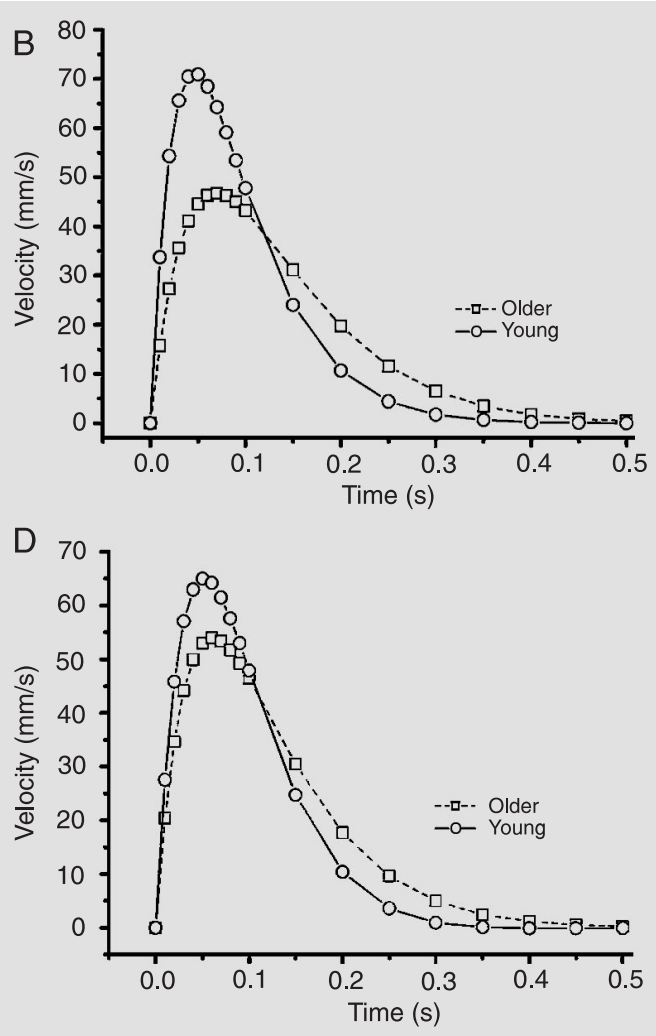
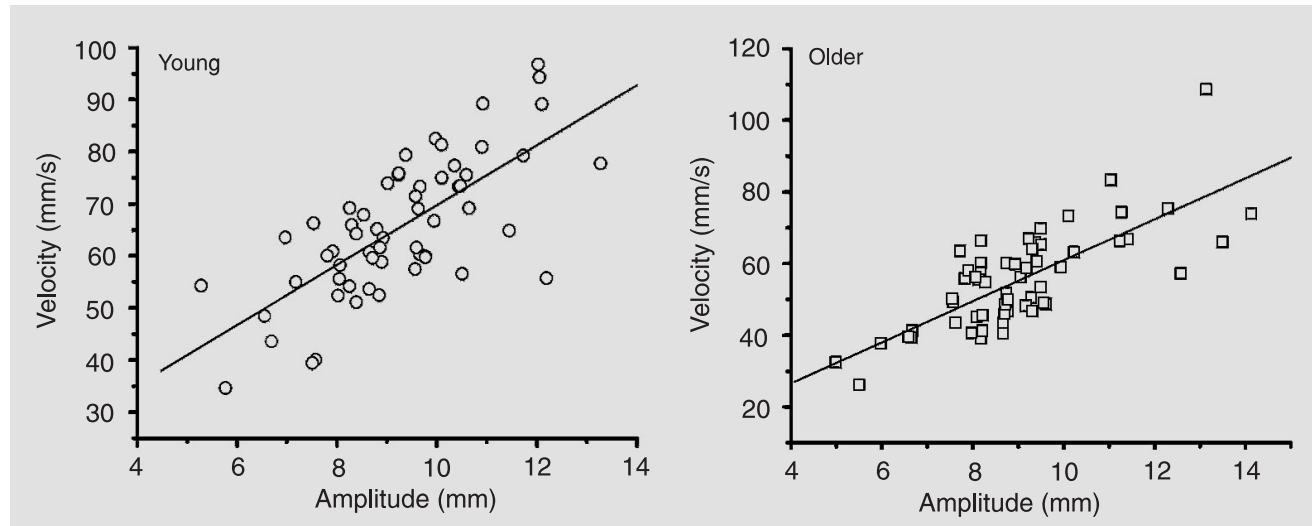

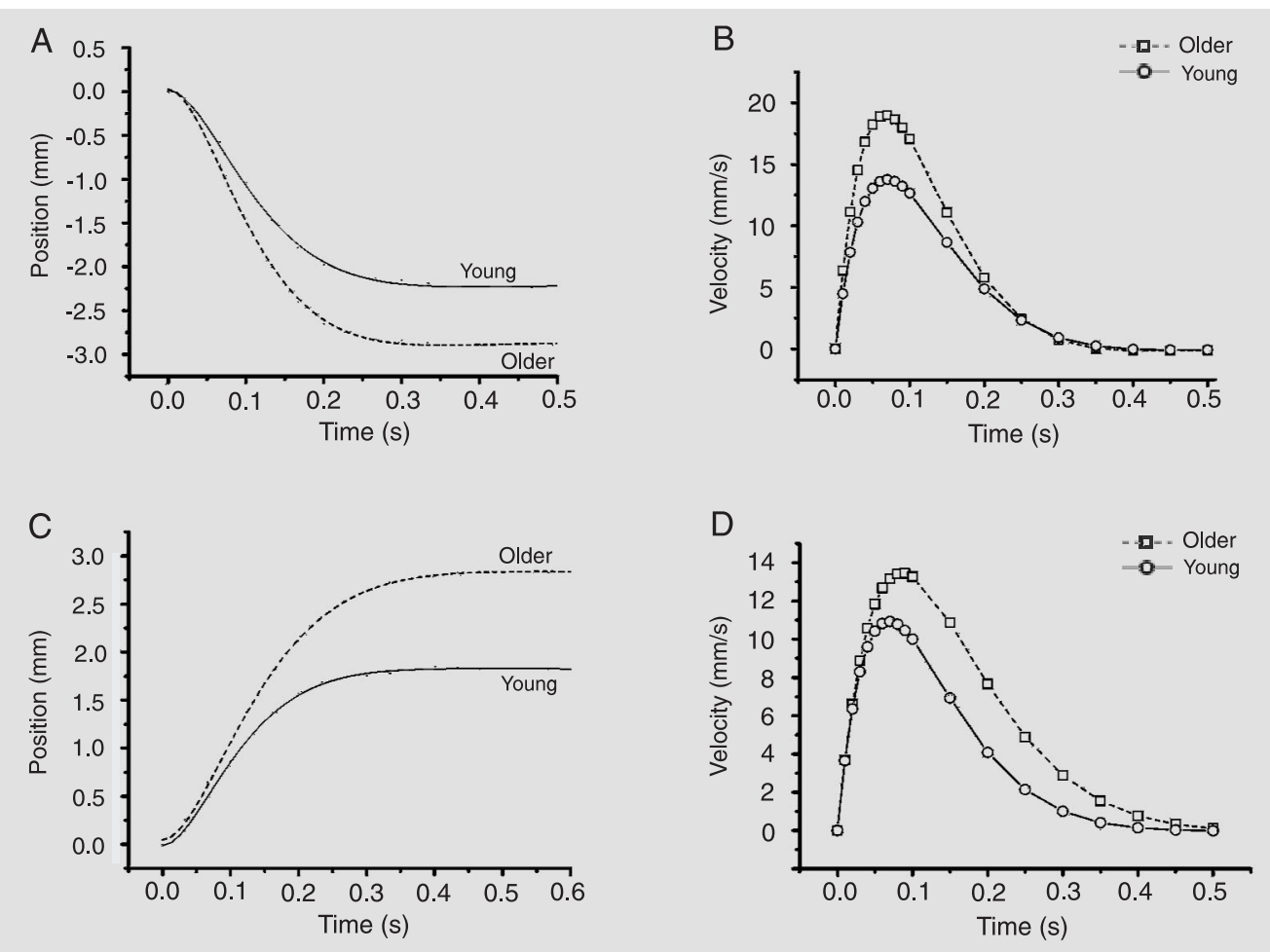
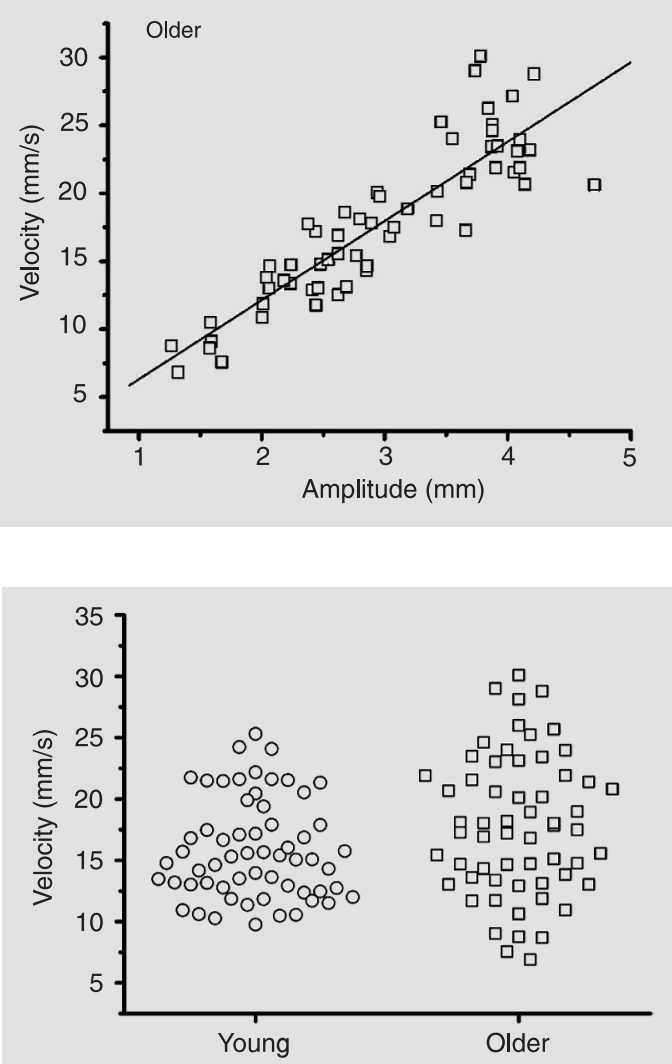

Figure 4. Lower eyelid saccade amplitude: $A$, down; $C$, up. The evolution of velocity with time ( $B$, down; $D$, up) is illustrated on the right. Note the difference in amplitude between the older and young subjects.

Figure 5. Linear relationships between lower eyelid saccade amplitude and velocity for young (left, $r=0.88$ ) and older subjects (right, $r=0.89$ ).

Figure 6. Distribution of lower eyelid saccade velocity in young and older subjects. 
and extends onto both the anterior and posterior surfaces of the tarsus (10).

Aging is also believed to induce attenuation of the lower eyelid retractors. The socalled reverse lower eyelid ptosis is characterized by a reduction of the distance from the lower eyelid margin to the pupil center (11). This tendency of age to induce lower eyelid ptosis was seen in our older subjects who presented a smaller mean mid-pupil eyelid distance than the young subjects.

The exact mechanism of transmission of active forces to the lower eyelids during downgaze is still obscure. In one study using dynamic high-resolution magnetic resonance imaging it was found that the length of the anterior capsulopalpebral fascia between the tarsus and inferior oblique muscle remained constant in downgaze (12). The authors concluded that the source of the tension of the lower eyelid was posterior to the capsulopalpebral fascia.

The forces involved in the upward displacement of the lower eyelid have not been investigated. Jones (9) suggested that the pull of the upper eyelid structures at the lateral canthus and the contraction of the lower preseptal muscle were the factors that explained the lower eyelid movement upwards. The lateral pull during upward eye saccades is easily demonstrated. In fact, when the direction of gaze shifts from down- to upgaze, the lateral canthus also moves up, increasing the palpebral fissure angle (13). However, contraction of the orbicularis muscle is unlikely to occur, because it has been shown that the orbicularis does not play a role during upper eyelid saccades (14).

If the capsulopalpebral fascia maintains its shape during the upgaze it seems more natural to consider that the key source of the lower eyelid movement is the rotation of the eye that pushes up the whole complex of orbital fat, inferior fornix and lower eyelid retractors. The fact that the amplitude of the lower eyelid saccades was greater in our older subjects suggests that the transmission of force to the lower eyelid is not abolished with age. We believe that the horizontal laxity which is clinically evident in the majority of elderly subjects is the factor that explains our findings. In fact, it seems reasonable to accept that if the eye rotation is the element that generates tension on the lower lid, a sagging lid would have more mobility than a tight one. In this conceptual framework, attenuation of the lower eyelid retractors could, in fact, contribute to higher mobility of the lid. It is possible that the lower eyelid retractors, besides acting as an element of transmission of forces to the lid in downgaze, also diminish the mobility of the lid in upgaze. Although none of the elderly subjects had entropion, we believe that our data support the investigators who stress the importance of correction of horizontal laxity as a key step in the stabilization of the lower eyelid of patients with involutional entropion (15).

\section{References}

1. Becker W, Fuchs AF. Lid-eye coordination during vertical gaze changes in man and monkey. J Neurophysiol 1988; 60: 1227-1252.

2. Castanares S. Classification of baggy eyelids deformity. Plast Reconstr Surg 1977; 59: 629-633.

3. Frueh BR. The mechanistic classification of ptosis. Ophthalmology 1980; 87: 1019-1021.

4. Dalgleish R, Smith JL. Mechanics and histology of senile entropion. Br J Ophthalmol 1966; 50: 79-91.

5. Wouters RJ, van den Bosch WA, Mulder PG, Lemij HG. Upper eyelid motility in blepharoptosis and in the aging eyelid. Invest
Ophthalmol Vis Sci 2001; 42: 620-625

6. Shore JW. Changes in lower eyelid resting position, movement, and tone with age. Am J Ophthalmol 1985; 99: 415-423.

7. Malbouisson JM, E Cruz AA, Messias A, Leite LV, Rios GD. Upper and lower eyelid saccades describe a harmonic oscillator function. Invest Ophthalmol Vis Sci 2005; 46: 857-862.

8. Dortzbach RK, Sutula FC. Involutional blepharoptosis. A histopathological study. Arch Ophthalmol 1980; 98: 2045-2049.

9. Jones LT. The anatomy of the lower eyelid and its relation to the cause and cure of entropion. Am J Ophthalmol 1960; 49: 29-36. 
10. Hawes MJ, Dortzbach RK. The microscopic anatomy of the lower eyelid retractors. Arch Ophthalmol 1982; 100: 1313-1318.

11. Bartley GB, Frueh BR, Holds JB, Linberg JV, Patel BC, Hawes MJ. Lower eyelid reverse ptosis repair. Ophthal Plast Reconstr Surg 2002; 18: 79-83.

12. Goldberg RA, Lufkin R, Farahani K, Wu JC, Jesmanowicz A, Hyde JS. Physiology of the lower eyelid retractors: tight linkage of the anterior capsulopalpebral fascia demonstrated using dynamic ultrafine surface coil MRI. Ophthal Plast Reconstr Surg 1994; 10:
87-91.

13. Alves LA, Cruz AAV, Souza ADA, Ruiz ES. Palpebral fissure changes during upgaze and downgaze. Invest Ophthalmol Vis Sci 1998; 39: 1011 (Abstract).

14. Evinger C, Manning KA, Sibony PA. Eyelid movements. Mechanisms and normal data. Invest Ophthalmol Vis Sci 1991; 32: 387-400.

15. Danks JJ, Rose GE. Involutional lower lid entropion: to shorten or not to shorten? Ophthalmology 1998; 105: 2065-2067. 